\title{
A Platform with Multiple Head-Mounted Displays for Advanced Training in Modern Driving Schools
}

\author{
Kareem Abdelgawad ${ }^{1, *}$ (i) , Jürgen Gausemeier ${ }^{1}$, Jörg Stöcklein ${ }^{2}$, Michael Grafe ${ }^{2}$, \\ Jan Berssenbrügge ${ }^{2}$ and Roman Dumitrescu ${ }^{2}$ \\ 1 Heinz Nixdorf Institute, University of Paderborn, 33102 Paderborn, Germany; \\ Juergen.Gausemeier@hni.upb.de \\ 2 Fraunhofer Institute for Mechatronic Systems Design IEM, 33102 Paderborn, Germany; \\ Joerg.Stoecklein@iem.fraunhofer.de (J.S.); Michael.Grafe@iem.fraunhofer.de (M.G.); \\ Jan.Berssenbruegge@iem.fraunhofer.de (J.B.); Roman.Dumitrescu@iem.fraunhofer.de (R.D.) \\ * Correspondence: Kareem.Abdelgawad@hni.upb.de; Tel.: +49-5251-606-228
}

Received: 22 September 2017; Accepted: 10 October 2017; Published: 13 October 2017

\begin{abstract}
Automotive manufacturers and suppliers develop new vehicle systems, such as Advanced Driver Assistance Systems (ADAS), to increase traffic safety and driving comfort. ADAS are technologies that provide drivers with essential information or take over demanding driving tasks. More complex and intelligent vehicle systems are being developed toward fully autonomous and cooperative driving. Apart from the technical development challenges, training of drivers with these complex vehicle systems represents an important concern for automotive manufacturers. This paper highlights the new evolving requirements concerning the training of drivers with future complex vehicle systems. In accordance with these requirements, a new training concept is introduced, and a prototype of a training platform is implemented for utilization in future driving schools. The developed training platform has a scalable and modular architecture so that more than one driving simulator can be networked to a common driving instructor unit. The participating driving simulators provide fully immersive visualization to the drivers by utilizing head-mounted displays instead of conventional display screens and projectors. The driving instructor unit consists of a computer with a developed software tool for training session control, monitoring, and evaluation. Moreover, the driving instructor can use a head-mounted display to participate interactively within the same virtual environment of any selected driver. A simulation model of an autonomous driving system was implemented and integrated in the participating driving simulators. Using this simulation model, training sessions were conducted with the help of a group of test drivers and professional driving instructors to prove the validity of the developed concept and show the usability of the implemented training platform.
\end{abstract}

Keywords: driver assistance systems; autonomous and cooperative driving; driving schools; driving simulators; multiple head-mounted displays; shared virtual environments

\section{Introduction}

Road safety and driving comfort are significant concerns in the automotive realm. Therefore, automotive manufacturers and suppliers develop new vehicle systems to reduce the driving stress or to support drivers in critical traffic situations. Advanced Driver Assistance Systems (ADAS) present an example of such new vehicle systems. ADAS are mechatronic systems that monitor the vehicle, driver's behavior, and the surrounding environment [1]. They deliver information about the surrounding traffic and carry out difficult driving tasks. Some ADAS alert drivers to inconvenient traffic situations. Other ADAS do more than recognize traffic situations and warn the drivers: they also intervene actively in order to prevent possible collisions. Diverse sensor technologies and decision 
algorithms are developed to provide different levels of assistance and automation [1]. Building on the rapid development of ADAS, highly automated and cooperative vehicle technologies are gaining considerable attention in the automotive world [2]. The training of drivers with these advanced vehicle systems represents a significant burden for automotive manufacturers.

The rest of this paper is organized as follows. Section 2 elaborates the problem and deduces the requirements of a new training concept for future driving schools. Section 3 introduces the concept of the proposed solution. Section 4 illustrates the architecture of the developed training platform and the underplaying implementation aspects of its main components. Moreover, the implementation of a simulation model of an autonomous driving system is presented as further development for the work provided in References [3,4]. Section 5 presents the test setup and the validation results. Finally, Section 6 outlines the conclusions, emphasizes the novelty of the presented training concept and platform, and reveals future work.

\section{Problem Description and Call for Action}

Driving simulators offer a practical means for the training of drivers in general [5]. Various driving schools use driving simulators to introduce basic driving tasks to the beginners. That is, the beginners can start in safe and controlled virtual environments before subjecting them to real field drives. They learn how to handle different situations that can be encountered in real traffic environments. A lot of simulation scenarios are designed to cover various aspects, such as pre-drive checks, traffic rules, and driving in hard weather conditions. Figure 1 shows two driving simulators utilized at the Ringhoff and Hainer driving schools in Germany for basic training purposes.

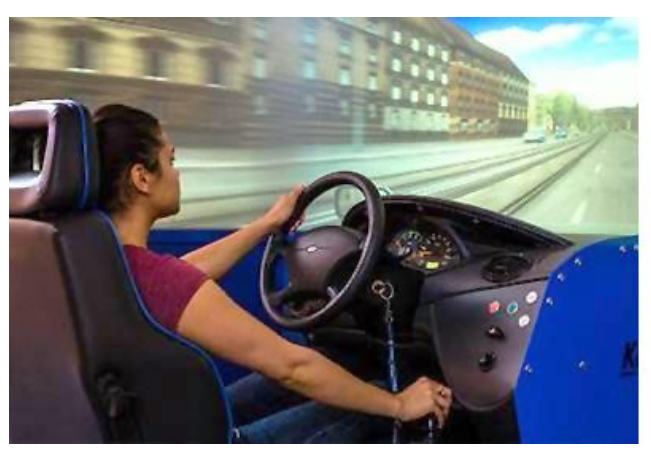

(a)

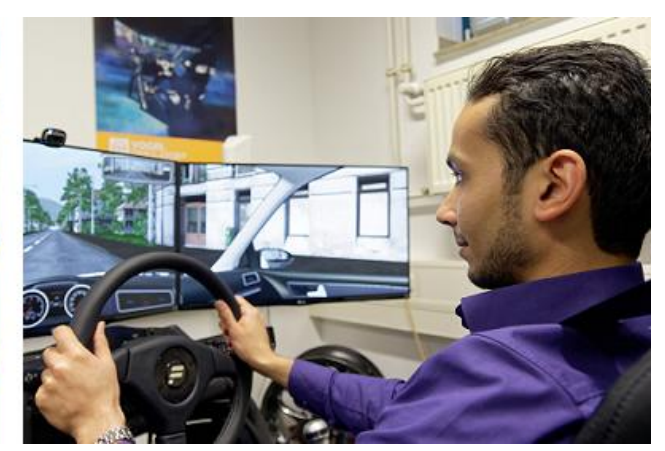

(b)

Figure 1. Utilization of driving simulators in driving schools in Germany: (a) Driving simulator at the Ringhoff driving school; (b) Driving simulator at the Hainer driving school.

The driving simulator shown in Figure 1a is utilized at the Ringhoff driving school in Paderborn, Germany. This driving simulator has adapted dashboard and driving instruments of a real passenger car (Ford, Dearborn, MI, USA) and a cylindrical visualization system (AGRONN simulation technologies, Istanbul, Turkey) that provides a horizontal field of view of 180 degrees. This driving simulator is used to make drivers familiar with the basic driving tasks, such as vehicle parking and lane change maneuvers, before driving in real traffic environments. The driving simulator shown in Figure $1 \mathrm{~b}$ is utilized at the Hainer driving school in Unna, Germany. This driving simulator (VOGEL, Munich, Germany) has a realistic driving platform and three wide screens that provide a horizontal field of view of 120 degrees. The driving simulator is used to conduct basic training sessions for beginners.

However, a lot of vehicles are currently equipped with various assistance systems. Some of these assistance systems are becoming obligatory according to the new traffic regulations. Training of drivers with these assistance systems represents a crucial concern to automotive manufacturers beside the technical development challenges. This particular concern contributes considerably to the sustainability 
of the automotive market. Specifically, the human-machine interaction is one of the main aspects related to the customer acceptance. This covers both the physical interface between drivers and assistance systems and the required understanding to operate these systems properly. If drivers are not well trained, these systems may have negative impacts with respect to drivers' behavior, reaction time, or situation awareness [6]. Problems may arise when drivers feel out of control, if there are many options, or if actions do not lead to the expected results. In addition to the associated safety issues and the lack of regulatory permissions, training of drivers with assistance systems in real traffic environments leads to constraining efforts and costs. Reference [3] provides a low-cost driving simulator that can be used for development and initial training purposes with simple driver assistance systems, such as, e.g., Lane departure warning. In Reference [4], a simulation framework is provided to develop and test more advanced driver assistance systems, such as, emergency brake and steering assistance systems. To address the topic in close cooperation with industry partners and driving schools, the transfer project inTraSim has been carried out at the Heinz Nixdorf Institute in Paderborn, Germany. The main objective of this project was the development of a driving simulator that can be utilized in driving schools to conduct training sessions with driver assistance systems. In addition to the Heinz Nixdorf Institute, the consortium of the project inTraSim consisted of Fahrerakademie Paderborn (modern training center for truck and bus drivers), Aerosoft $\mathrm{GmbH}$ (provider of advanced software packages for the realistic simulation of vehicles and airplanes), and VDL Bus \& Coach GmbH (manufacturer of buses and chassis modules, Büren, Germany). Figure 2 shows the driving simulator developed within the project inTraSim at the Heinz Nixdorf Institute.

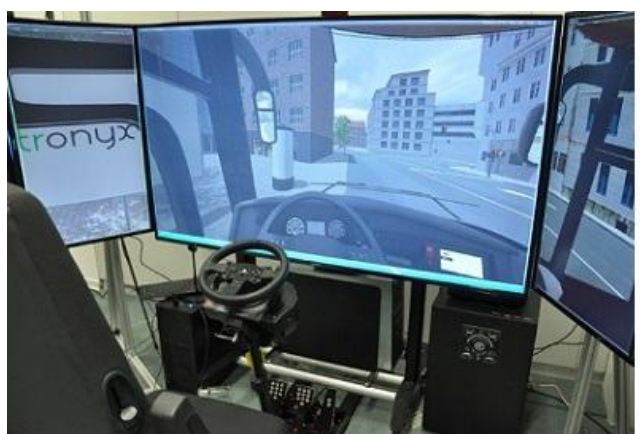

(a)

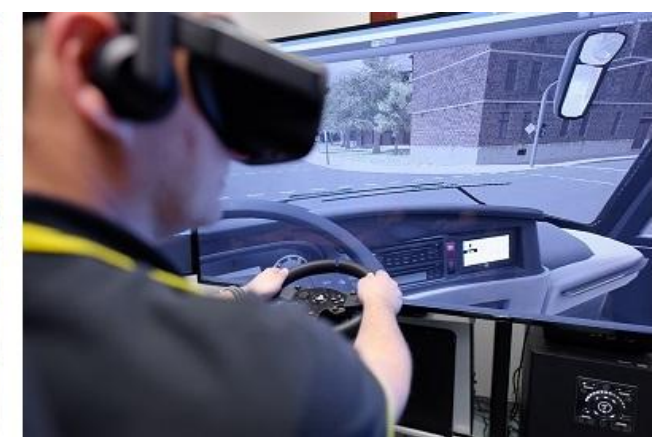

(b)

Figure 2. A driving simulator developed within the project inTraSim: (a) The hardware equipment of the developed driving simulator; (b) A test driver performing a turn-right maneuver.

As shown in Figure 2a, the developed driving simulator has a real bus driving seat and is equipped with three front screens to cover a horizontal field of view of 120 degrees. Figure $2 b$ shows a bus test driver that intends to turn right with the help of a blind spot assistance system. Other driver assistance systems can be introduced to bus drivers using this driving simulator, such as cruise control and lane departure warning.

However, a considerable part of the driving responsibility will be carried out by more intelligent vehicle systems in the future, whereas drivers will gain more supervisory roles. The complexity of such systems can introduce more cognitive loads on drivers and negatively affect system acceptance [6]. Hence, a new concept for the utilization of driving simulators at driving schools is required to keep up with the rapid advancements of future vehicle systems. In particular, the following demands have been derived at the end phase of the transfer project inTraSim. The following aspects represent the necessary capabilities and properties of a pursued new training system:

- Simultaneous training sessions

As it is expected to conduct extensive and time-consuming training sessions with advanced vehicle systems, the training system should allow the participation of several drivers simultaneously. 
- Feasible and cost-effective solution

The entire training system should remain cost-effective for driving schools. That is, it should not depend on expensive simulator components. Moreover, the training system should impose only feasible space requirements.

- $\quad$ Training with advanced vehicle systems

The training system should support the training with advanced vehicle technologies, such as highly and fully automated assistance functions.

- More immersion and engaging virtual scenes

Realistic virtual replicas of the vehicle interior, user interface, and surrounding traffic participants should be provided by the training system to increase immersion and ensure engaging training sessions for drivers.

- Interactive supervision and instruction of drivers

The training system should provide a capability for close accompanying supervision and the instruction of trainees. Instructors at driving schools still should be able to carry out their usual supervisory and instruction roles.

The demand for these five particular aspects has been confirmed during the workshops held with instructors from the driving schools Hainer and Ringhoff in Germany. The instructors of these driving schools pointed to the necessity of more effective and modern solutions for the training of drivers. According to the literature review and the solid expertise in this field, no driving simulation platforms to date support all the aforementioned aspects for the practical utilization in driving schools. The following section provides the proposed solution to fulfill the aforementioned upgrade demands of training systems at future driving schools.

\section{Proposed Concept and Solution}

This work provides a new concept to conduct training sessions with advanced vehicle systems at modern driving schools. The concept considers the particular system requirements presented in the previous section. Specifically, the proposed solution introduces a training platform that consists of several driving simulators that are networked to a central instructor unit. That is, several drivers can participate in the training sessions simultaneously in response to the first system requirement. Figure 3 shows a $3 \mathrm{D}$ layout for the proposed training platform.

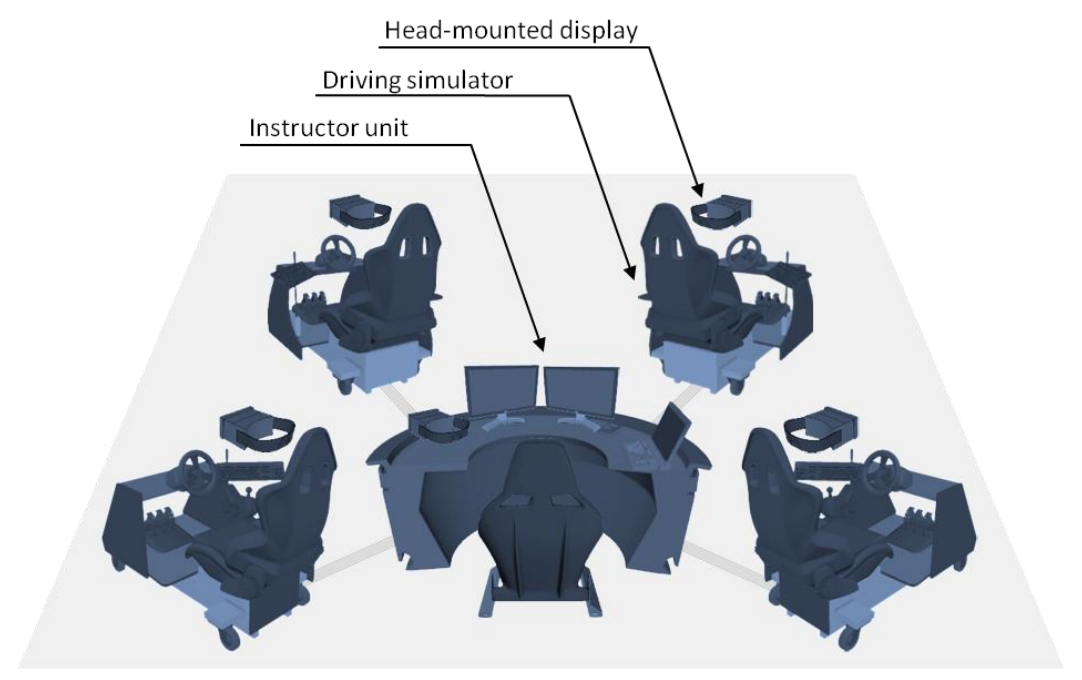

Figure 3. Layout of a training platform with multiple head-mounted displays for modern driving schools. 
Motion platforms consume a major part of the available budget and require considerable space, unlike other driving simulator components. Moreover, the operation costs of driving simulators increase substantially if motion platforms are utilized due to the associated huge power consumption. Therefore, the participating driving simulators of the proposed training platform have no motion platforms in response to the second system requirement. However, the driving simulators are equipped with speakers and powerful subwoofers to produce sensible road noise and deliver slight vehicle vibration [7]. Even without motion platforms, this allows drivers to experience a good extent of realistic dynamic aspects, such as accelerating/deceleration and driving over different road surfaces. The participating driving simulators are equipped with simulation models for various advanced vehicle systems, such as, e.g., an autonomous driving system. This capability comes in response to the third system requirement. Moreover, the participating driving simulators are equipped with Head-Mounted Displays (HMDs) as the main visualization systems [8]. These are 3D interactive displays that provide full 3D-viewing and deliver user-dependent scenes. Modern versions of HMDs do not only allow free head motion, but also provide a good extent of body mobility [8]. Drivers can freely move their heads and marginally change their body position without losing the engagement with the displayed virtual scenes. This particular characteristic is necessary for training with advanced vehicle systems that require full immersion within the virtual driving environment. The utilization of HMDs fulfills the fourth system requirement concerning the engagement of the conducted training sessions. In addition, current rapid advancements in the field of virtual and augmented reality pushed the modern HMDs to be cheaper and have lighter weights. Currently available HMDs have low cost and less space requirements in comparison to traditional display systems, such as, e.g., screens and projectors. These latter characteristics contribute to the fulfillment of the second system requirement with respect to system costs and practical utilization. The instructor unit consists of a computer with a software tool for centralized session control, monitoring, and evaluation. This unit is equipped with an additional HMD for the driving instructor to step into the virtual driving scenarios of the drivers if necessary. That is, the same virtual environment can be shared between two head-mounted displays simultaneously. Hence, the typical and necessary supervisory role of instructors at driving schools is still provided using the presented training platform. This distinguished capability of the proposed training platform fulfills the fifth system requirement.

\section{System Architecture}

A consistent architecture for the training platform has been designed to realize the proposed concept. Particular focus during the composition of this architecture is given to the modularity and reconfigurability system design principles. Figure 4 shows the architecture of the developed training platform and its main building components.

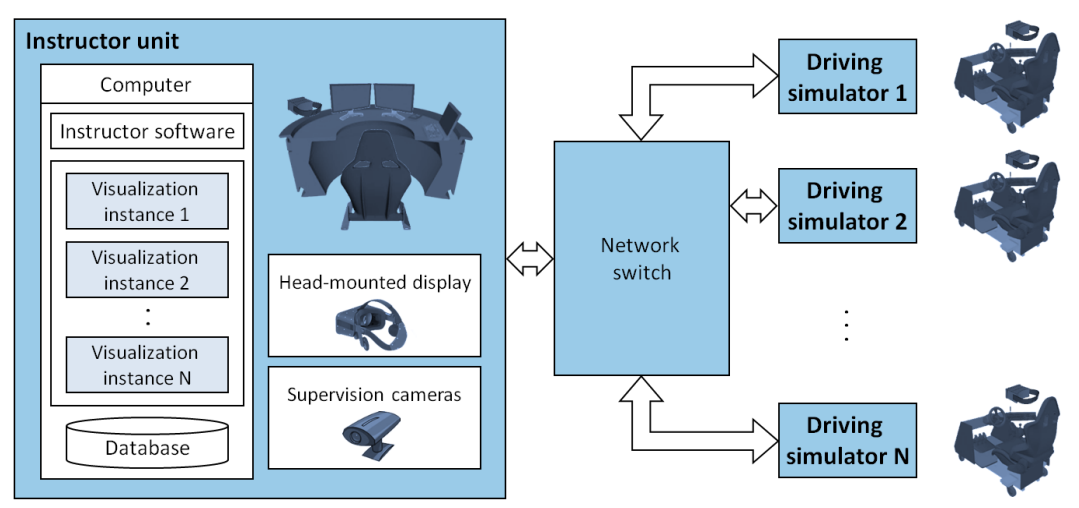

Figure 4. Architecture of the developed training platform with multiple head-mounted displays. 
The instructor unit represents the central component within the shown system architecture. This unit includes mainly a computer with a software tool used by the session instructor. In addition to the instructor software tool, there are instances of the visualization software of each of the participating driving simulators installed on the computer of the instructor unit. Through a dedicated local network, each driving simulator sends the position and orientation information of its simulated vehicle and those of the traffic participants to the instructor unit. In addition, the main signals of each simulated vehicle, such as, e.g., steering wheel angle, acceleration and brake pedals, and gear state, are sent to the instructor unit. Specifically, this data is forwarded to the corresponding visualization instances to animate the scenes delivered to the head-mounted display of the instructor. By selecting a particular trainee through the instructor software tool, the instructor can monitor the vehicle signals and switch between different views of the driving scenario. Moreover, a supervision camera is directed to each participating driving simulator. These cameras send video data to the instructor unit, so that the instructor can observe the gaze behavior of any selected trainee. In the presented architecture, the driving simulators are networked to the central instructor unit in a loosely-coupled fashion. That is, the simulation software packages of these driving simulators have no particular knowledge about the internal operation of the instructor software tool. This characteristic adds a major scalability advantage to the training platform. It is convenient to integrate different driving simulators to the training platform, provided that instances of their visualization software are installed on the computer of the instructor unit. Figure 5 shows a prototype of the training platform that was implemented in accordance with the presented system architecture.

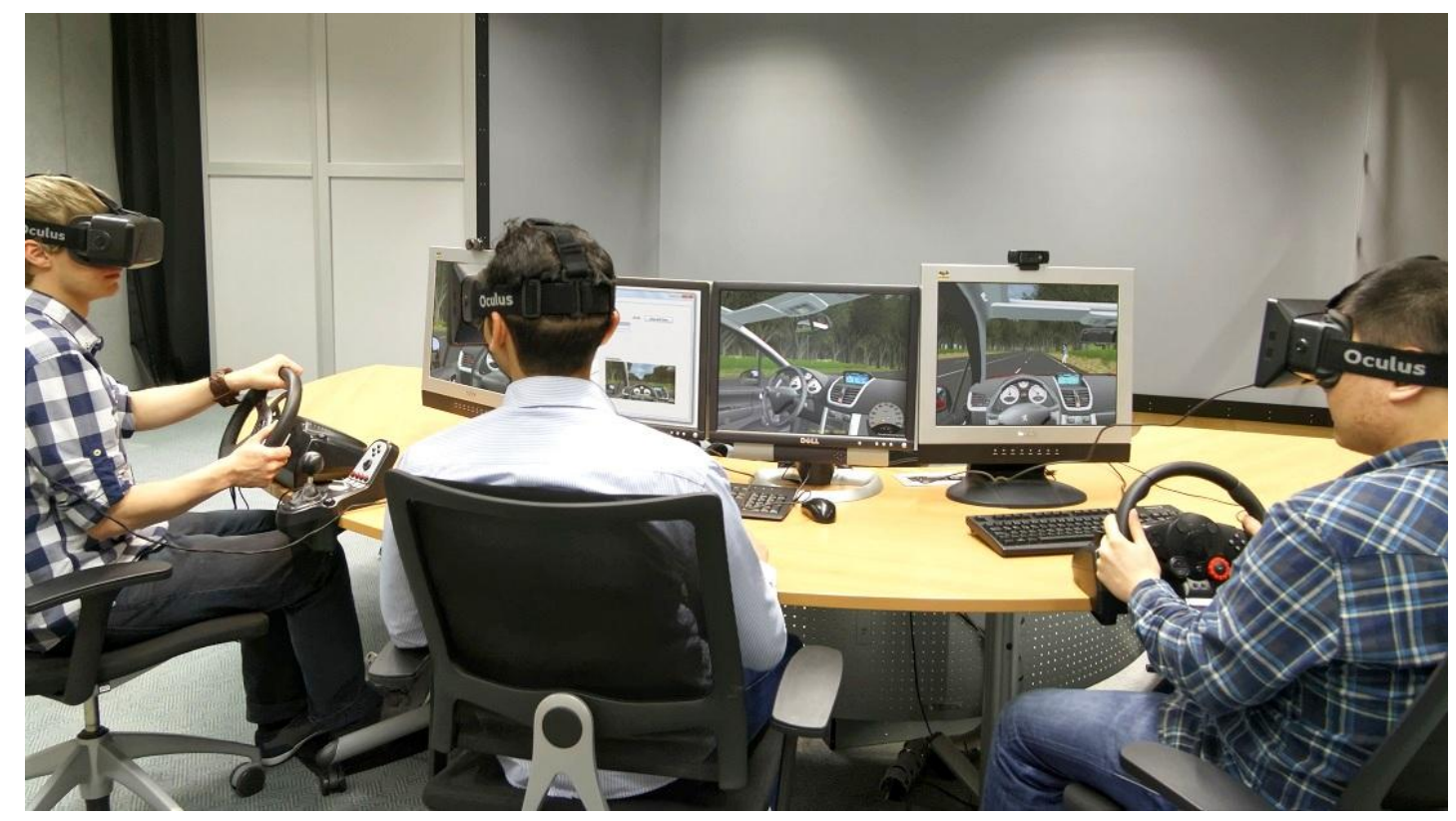

Figure 5. A prototype of the proposed training platform with multiple head-mounted displays.

In its current implementation, the developed training platform involves two similar driving simulators connected to the instructor unit. Apart from the hardware, no commercial or off-the-shelf programs are used in these driving simulators. The software packages of these driving simulators have been developed fully at the Heinz Nixdorf Institute. The following subsection discusses the main building blocks of these software packages. A simulation model of an autonomous driving system is discussed as further development for the work presented in References [3,4]. 


\subsection{Utilized Driving Simulators}

Two PC-based driving simulators without motion platform were configured and integrated within the instructor unit [3]. Each driving simulator has a commercial wheel-transmission-pedals set that provides both low-cost and reasonable physical feedback and control cues. A commercial head-mounted display is utilized in this work instead of conventional simulator display systems based on the requirements derived in Section 2. Figure 6 shows the main building components of one of these driving simulators.

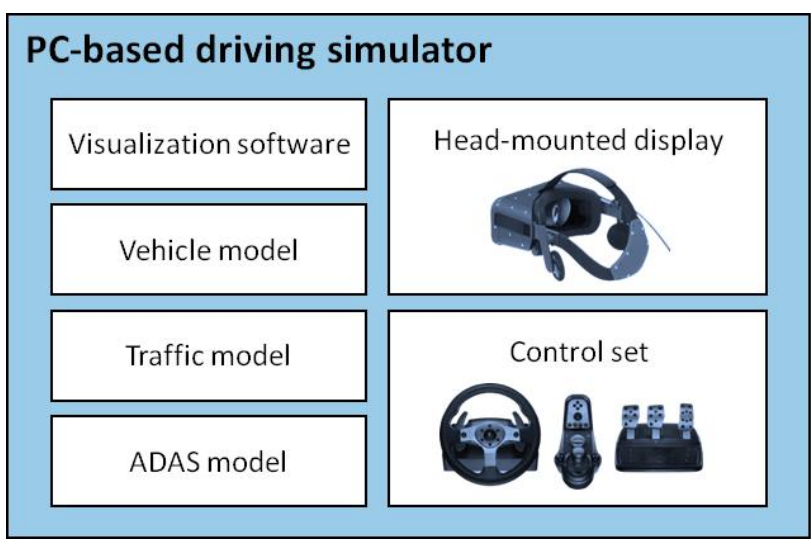

(a)

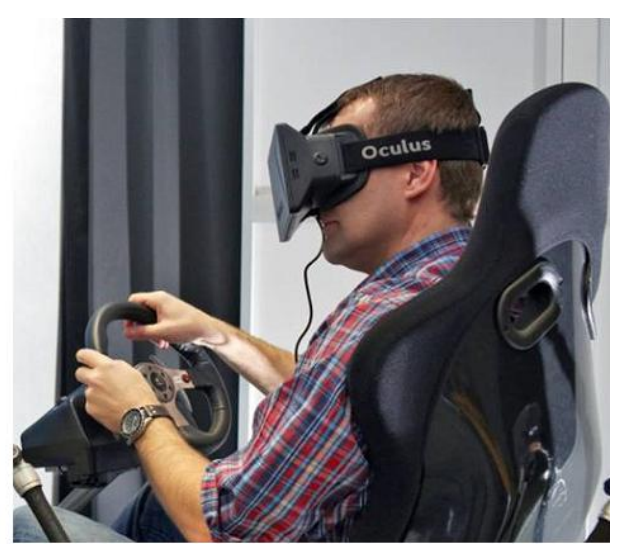

(b)

Figure 6. The integrated and further developed driving simulator: (a) The internal structure of the driving simulator; (b) A test driver experiencing virtual scenes using a head-mounted display. ADAS: Advanced Driver Assistance Systems.

Four main software models constitute the overall simulation environment of the PC-based driving simulator as shown in Figure 6a. With this particular modular structure, the modification of the models or their interfaces does not require considerable effort. That is, each of the models can be developed further separately or exchanged without deep knowledge of the implementation of the other models. The following subsections present the underlying implementation aspects of each model and the added modifications in this work to fulfill the requirements presented in Section 2. More detailed information about the implementation and a comprehensive discussion about the input-output relationships of these models are provided in References [3,4].

\subsubsection{Visualization Software}

The visualization software represents the main feedback cue of the driving simulator. It was developed with Unity3D (Unity Technologies, San Francisco, CA, USA) [9]. Realistic 3D models for the main simulated vehicle and the surrounding traffic participants were built in the visualization environment. Moreover, real highways and city streets can be generated; this is necessary for an engaging and realistic training of drivers. The drivers can experience different weather conditions, such as, e.g., snow, fog, rain, etc. Figure 7 shows sample screen shots of the developed 3D environment for day- and nighttime driving conditions. 


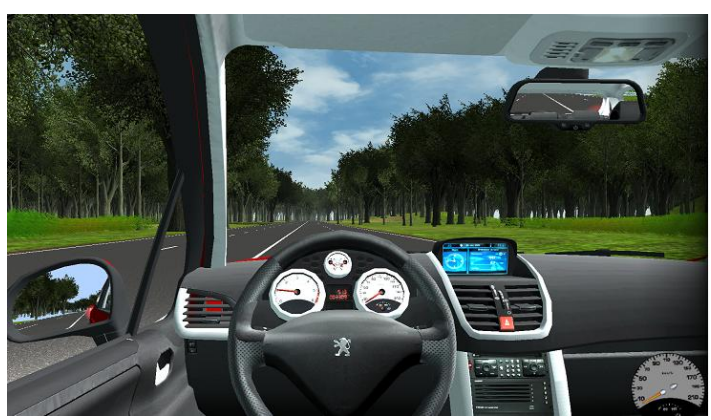

(a)

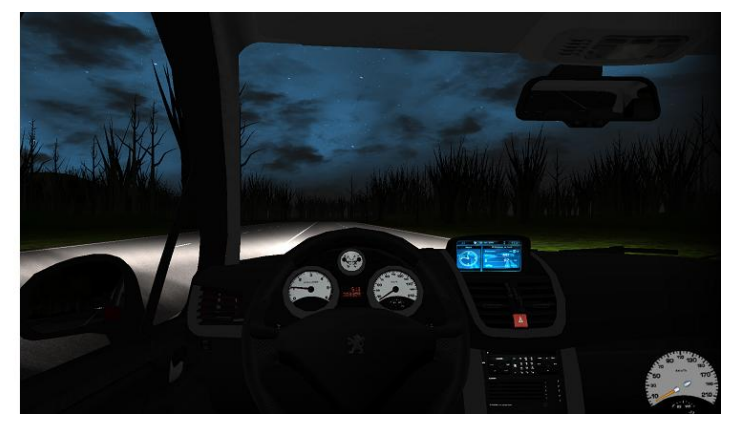

(b)

Figure 7. Sample screen shots for the 3D environment: (a) Daytime driving; (b) Nighttime driving.

In addition, the 3D models are accompanied with realistic sound effects to provide reasonable acoustic feedback cues. The visualization software has been developed further in this work to enable the use of head-mounted displays. To present realistic vehicle dynamics and traffic interactions, the $3 \mathrm{D}$ models receive the position and orientation data from the vehicle physics and traffic simulation models that are presented subsequently.

\subsubsection{Vehicle Physics Simulation Model}

Realistic modeling of vehicle dynamics is necessary for driving simulation in general. The utilized vehicle model and the rest of models presented subsequently in this work were implemented with MATLAB/Simulink (MathWorks, Natick, MA, USA). The vehicle model reproduces the real physical characteristics of the main simulated vehicle and provides 14 Degrees Of Freedom (DOF) [10]. A nonlinear double-track model is used to reproduce the horizontal vehicle dynamics. This model provides 3 DOF: a rotational motion around the road vertical direction, as well as longitudinal and lateral translational motions. In the double-track model, the longitudinal and lateral velocities, as well as the yaw rate of the simulated vehicle, are deduced by a set of differential equations that use Newton's law of motion and fundamental geometrical relationships [10].

The vertical dynamics of the vehicle depend on suspension units fixed at each wheel of the vehicle. The vehicle chassis is connected to four wheels through these suspension units. Each suspension unit is represented as a simple mass-spring-damper model [10]. The suspension units are connected through primary geometrical relationships. Each wheel has a vertical translational motion and a rotational motion around its lateral axis. Moreover, each of the front wheels has a rotational motion around its vertical axis. In addition to the horizontal and vertical dynamics, sub-models for the gearbox, engine, tires and wheels, differential, steering, and braking supplement the vehicle physics simulation model.

\subsubsection{Traffic Simulation Model}

The traffic model is used to simulate the surrounding programmed vehicles and the road. It simulates realistic interactions of the traffic vehicles. This is necessary to give reasonable feedback cues to the driver. Moreover, training with ADAS depends on the simulation of other road participants. In particular, the traffic simulation model consists mainly of four sub-models: driver model, road model, models of traffic vehicles, and a scenario manager. The traffic model receives the position, orientation, and speed of the main simulated vehicle from the vehicle physics simulation model; these are used mainly by the driver model to arrange for appropriate traffic flow.

The main task of the driver model is to achieve the desired traffic scenario without vehicle collisions. The road model is responsible for two major tasks. The first task is to transform the simulation local coordinate system $(\mathrm{s}, \mathrm{t})$ to the global coordinate system $(\mathrm{x}, \mathrm{y})$ understood by the visualization software. The position of each object within the physics simulation environment is defined relative to road local coordinate system. However, the visualization software defines each 
object in 3D simulation environment relative to a global coordinate system. The second major task of the road model is to define the friction and height of each point of the road. The height values are required by the visualization software to position the objects appropriately within the 3D simulation environment. The friction and height values are together required by the vehicle physics simulation model; they are used to calculate the horizontal and vertical vehicle dynamics respectively.

Each traffic vehicle model consists of two sub-models: the longitudinal direction vehicle sub-model and the lateral direction vehicle sub-model. The longitudinal direction sub-model receives the desired s-speed from the driver model. It calculates the actual s-speed with a smooth transition, which results from a combination of a simple second-order system and a P-controller. The actual s-position of the traffic vehicle is then calculated by integrating the actual s-speed. Similarly, the lateral direction sub-model receives the desired t-position from the driver model. It calculates the actual t-position with a smooth transition, which results from a combination of a simple second-order system and a P-controller. The idea of the traffic vehicle model is to produce smooth and realistic, i.e., no abrupt movements for the traffic vehicles. This is achieved through the transitional response of the second-order system to unit step inputs of the driver model. The traffic vehicles have to follow the predetermined longitudinal speed and lateral position given by the driver model. The scenario manager is used for arranging specific traffic situations, such as, e.g., a sudden vehicle incursion from right. It observes the position and speed of the main simulated vehicle and moves the traffic vehicles according to a desired predefined scenario. According to the simulated vehicle systems or functions, arbitrary different traffic scenarios can be added to this model. The driver model receives the vehicle positions and speeds determined by the scenario manager model. According to the current traffic situation, the driver model decides whether to execute the orders of the scenario manager or to override them. Switching between the different scenarios can be performed during simulation runtime.

\subsubsection{ADAS Simulation Model}

The design of the ADAS simulation model allows the integration of various driver assistance systems. This simulation model is composed of four sub-models: user interface, sensors model, controller module, and decision unit [4]. The user interface model receives signals from the hardware control set to handle the states of different ADAS. The sensors model consists of sub-models for long-range radar and short-range radar. In addition, it contains a simple camera model that resembles the road geometrics implemented within the traffic simulation model.

The controller module encloses mainly a longitudinal controller and a lateral controller. The longitudinal controller is based on a cascaded speed-acceleration simple control system [11]. A Proportional-Integral (PI) speed controller represents the outer loop of the longitudinal controller. The speed controller calculates the accelerations required to obtain the desired speed values. The desired accelerations are given to the acceleration controller that represents the inner loop of the longitudinal controller. The acceleration controller implements the inverse model of the vehicle dynamics and drivetrain [12]. The whole longitudinal controller exports the throttle angle or braking value to the vehicle model. The lateral controller carries out a path following control problem, i.e., how to control the main vehicle so that it can exactly follow a prescribed desired path [13]. This controller consists mainly of two further sub-models. By receiving a desired trajectory, a path following sub-model deduces the front axle force necessary to adjust the vehicle orientation. The path following sub-model utilizes a feedback linearization control method [13]. A steering calculation sub-model determines the steering angle corresponding to the desired lateral force. The lateral controller forwards the steering wheel angle necessary to guide the vehicle in the desired direction to the vehicle physics model. The designed longitudinal and lateral controllers can be used to realize a variety of ADAS functions.

The decision unit contains the logic of different ADAS in form of sub-routines. It observes the intention of driver through the inputs received from the hardware control set. The decision unit also monitors the state of the main simulated vehicle calculated by the vehicle physics model, i.e., 
position, orientation, and speed of the vehicle. Moreover, it gets the objects detected and filtered by the sensors model. According to the logic of the concerned ADAS function, a sub-routine determines whether the driver's requests or the logic of the ADAS function shall dominate in a particular driving situation. Sub-routines for emergency brake assistant and emergency steer assistant were developed and presented in a previous work [4]. However, the general design of the controller module and decision unit makes it convenient to develop and integrate new ADAS functions.

Autonomous vehicles are able to drive themselves as long as the input requirements of their logic are met [14]. When an autonomous driving system reaches the limit of its capabilities, it has to disband or hand over the control to the human driver. Taking full control back from an autonomous system has to be trained for and understood to avoid negative safety effects. A model for an autonomous driving system has been implemented in this work. It is used later as a validation example for the developed training platform. Autonomous driving presents a good example for advanced vehicle systems that drivers must learn before its deployment in real traffic environments. Specifically, it must be clear who is in control at each moment, i.e., the human or the automated driver. A separate sub-routine was implemented within the decision unit of the ADAS simulation model. Figure 8 shows a flow chart for a simplified version of the autonomous driving logic.

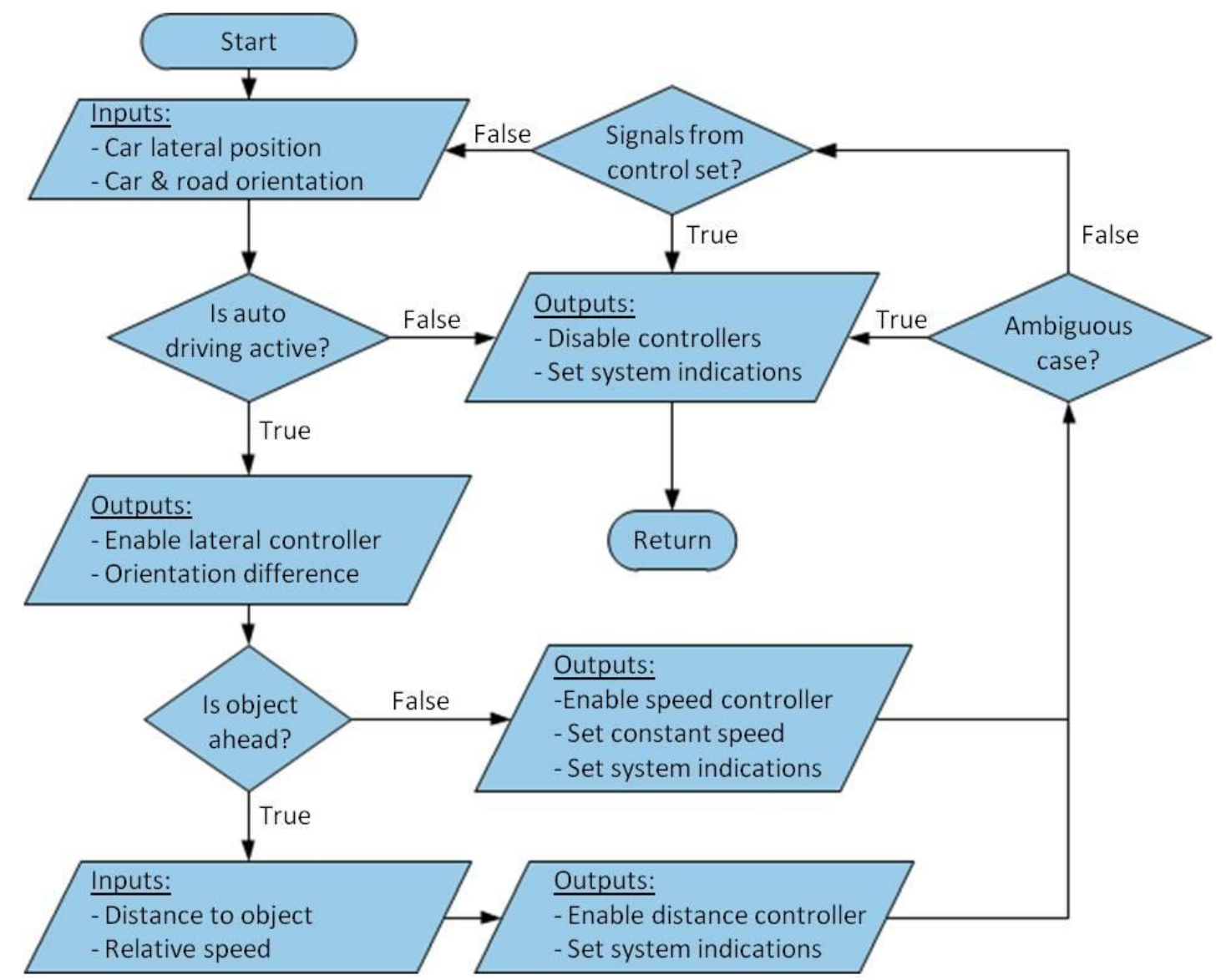

Figure 8. Simplified logic of the developed autonomous driving simulation model.

Upon activating the autonomous driving system, the sub-routine reads the current lateral position and orientation of the vehicle, as well as the road heading angle. A signal to activate the lateral controller is sent together with the difference heading between the vehicle and the road. Accordingly, the lateral controller adjusts the orientation of the vehicle. Moreover, the sub-routine checks whether a relevant object is detected in front of the vehicle by the sensors model. In case of a detected object, the longitudinal controller is enabled to maintain a constant distance to the object. Otherwise, it 
maintains an arbitrary constant speed. The sub-routine checks whether there is an ambiguous case. An ambiguous case can be programmed arbitrarily so that it is invoked at certain time points or situations. The ambiguous case is meant to simulate the capability boundary of real autonomous driving systems, such as, e.g., bad weather conditions. If it occurs, the sub-routine deactivates the lateral and longitudinal controllers. Moreover, it invokes optical and acoustic feedback so that the human driver takes over the control. Additional disband measures can be added arbitrarily at this point. For example, a braking maneuver can be applied to bring the vehicle to a safe state. The sub-routine monitors the signals from the control set continuously, i.e., the acceleration and brake pedals, as well as the steering wheel, to check the eventual preference of the human driver to gain the control at any moment.

Different technological capabilities for autonomous driving systems are being developed by different manufacturers. The logic of the autonomous driving system presented in this work is generic. That is, it has an initial set of capabilities and can be adapted to resemble different real systems. The following section presents the design of the instructor software tool and its capabilities.

\subsection{Instructor Software Tool}

The instructor software tool resides on the computer of the instructor unit. It was developed with MATLAB/GUI and designed so that it enables the instructor to control, monitor, and evaluate the training sessions. The instructor software tool consists of three main modules as illustrated in Figure 9.

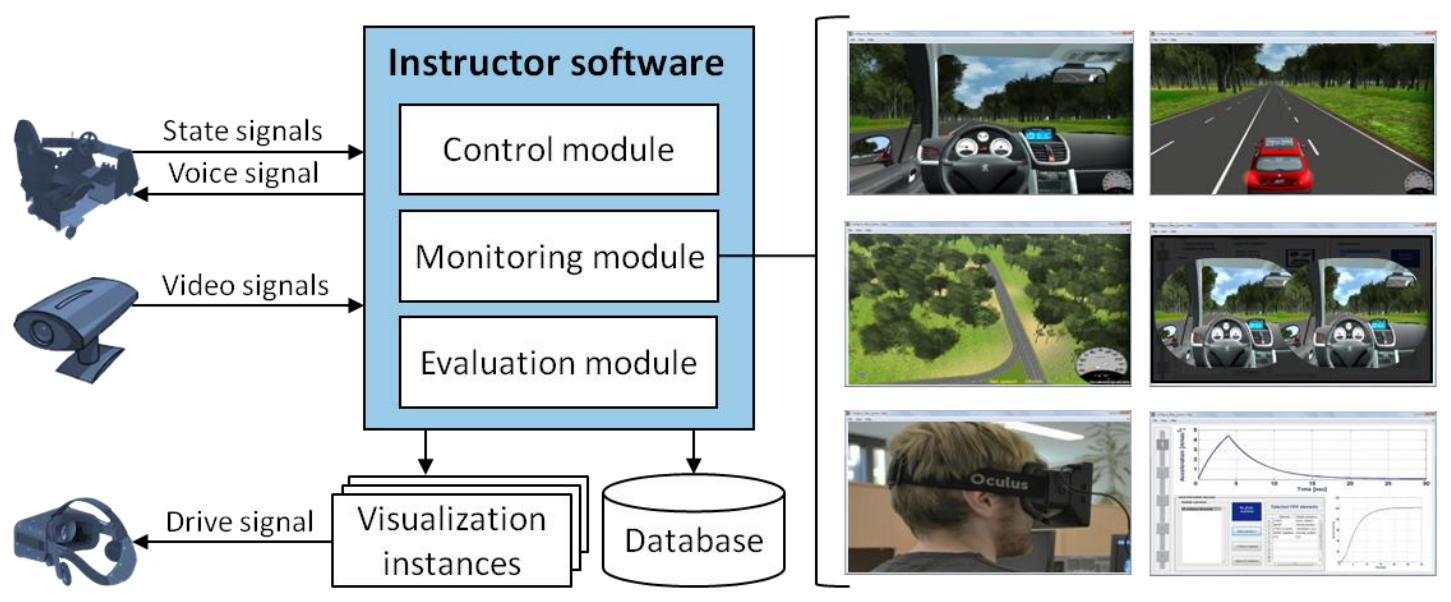

Figure 9. Structure and main input/output signals of the instructor software tool.

The instructor software tool receives the position and orientation information of the main simulated vehicle and the traffic participants from each participating driving simulator. Moreover, signals regarding the state of the vehicle and the assistance systems are received. In addition, the supervision cameras directed to each driving simulator send video signals to the instructor software. The following sub-sections discuss the concept of each module within the instructor software tool.

\subsubsection{Control Module}

The control module allows the instructor to create user profiles. Different data items, such as name, gender, age, and license type, can be registered. The control module is connected to a database, where users' data is saved. The instructor can view and edit the saved user profiles. Additionally, the instructor can start/stop or reset the training session of a selected trainee through the control module.

\subsubsection{Monitoring Module}

The monitoring module allows the instructor to select a particular trainee and switch between different perspectives of the driving situation as shown in Figure 9. It forwards the signals coming from 
a selected driving simulator to the corresponding visualization instance. The concerned visualization instance in turn drives the head-mounted display of the instructor unit, so that the instructor can step into the virtual environment of a selected trainee. Moreover, the instructor can monitor various signals regarding the state of a selected vehicle, such as steering wheel angle, acceleration and brake pedals, gear state, ADAS functions state, etc. Through the video signals delivered by the supervision cameras, the instructor can observe the gaze behavior of the selected trainee. This gives crucial indications about the traffic situation awareness [6].

\subsubsection{Evaluation Module}

The evaluation module saves the state signals of each simulated vehicle in a database. The data is sorted in sessions, which are associated to users' profiles created through the control module. The instructor can select a particular session and review the signal graphs. Maintaining these records allows the instructor to perform after-action analyses and discuss the results with the trainees.

\section{Results and Validation}

In general, there are mainly two approaches when it comes to the validation of interactive driving simulation: physical validation and behavioral validation [15]. The physical validation approach is concerned with parameters related to the simulated vehicle, such as the steering performance of the simulated vehicle in comparison to that of a real vehicle. The behavioral validation approach concentrates on the assessment of reactions and performance of drivers within the virtual traffic scenario. To show the usability and estimate the user acceptance of the developed training platform, this work considers specifically the behavioral validation approach. There are two types of measureable variables in this regard: physiological signs and driving performance [15]. Heart rate, respiration rate, and muscle tension are examples for the physiological signs. The driving performance can be measured, for example, by observing the ability to control the simulated vehicle appropriately or the interaction with other traffic participants [16]. As physiological signs are difficult to measure without dedicated special instruments, the developed training platform is validated by observing the driving performance of test persons [15]. Reaction time is one significant and relevant factor, which can be used to reflect the driving performance, especially when it comes to the utilization of driver assistance systems. The main purpose of the training platform is to introduce advanced vehicle systems to the trainees while not affecting their traffic situation awareness and avoiding eventual capability overestimation. Hence, the utilization of the training platform must lead to a considerable reduction in the reaction time of the trainees in response to the changing states of the advanced vehicle systems during driving. To that end, five test persons were involved in the behavioral validation process using the developed training platform under particular consideration of the reaction time. The group included persons of ages between 25 and 30 years old. While the involved persons did not have prior experience with driving simulators, they have similar experience levels with vehicle driving. In addition to the test persons, an experienced driving instructor was involved to carry out the supervision and instruction roles. The autonomous driving system was introduced to the test drivers. That is, they know in advance about the features of the system and how they should respond to its warnings and indications. The test persons drove in scenarios with destined and random ambiguous driving conditions, where the autonomous driving system gave warning signals and disbanded the control. Each driver had to drive three rounds. The reaction time with which the test drivers took over the control was measured automatically within the simulation model. However, the test drivers were not informed in advance that the reaction time is the main measurement of the experiment. This was particularly necessary in order not to affect the validation process by the awareness of the test drivers. Figure 10 shows the measured reaction times of the five test drivers after the three driving rounds. The measured reaction times have been rounded to the nearest tenth for presentation convenience. 


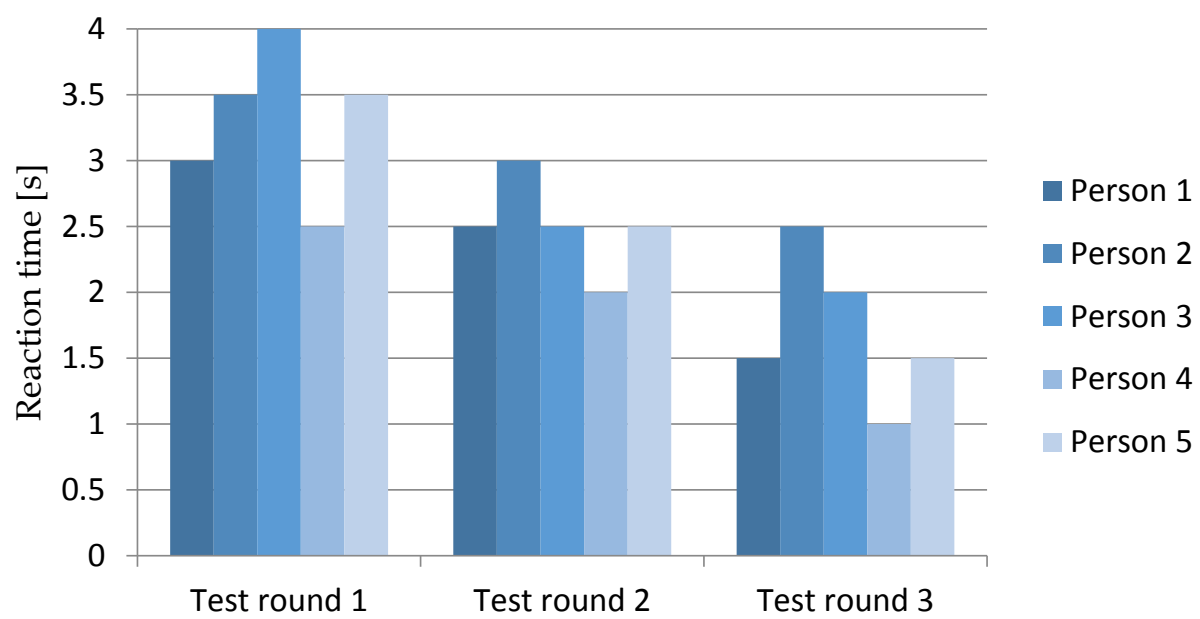

Figure 10. Reaction times of five test persons during three test drive rounds.

The reaction times of the test drivers in the first driving rounds were significantly longer than those of the subsequent driving rounds. The behavioral validation process proved certainly that the test drivers could respond to the warnings and indications of the autonomous driving system, i.e., they could take over the control with decreasing reaction times, as shown in Figure 10. The reaction times of all test drivers decreased throughout the three test drive rounds. Moreover, the involved driving instructor acknowledged the definite convenience and effectiveness of the developed training system. The following section derives the conclusions and announces future work to increase the capabilities of the developed training platform.

\section{Conclusions and Future Work}

Due to their safety and comfort features, advanced vehicle technologies such as ADAS attract considerable attention from both consumers and manufacturers. However, training drivers with these complex systems must be taken into consideration alongside their development and test challenges. Current driving schools utilize only conventional driving simulator solutions for training with basic driving tasks. The training platform developed in this work offers a practical solution for the safe and effective training of drivers in modern driving schools in accordance with the future vehicle systems. The novelty of this work can be summarized in the following three concrete aspects:

- Driving instructors can participate within the virtual environments of the drivers. Thereby, the typical and necessary supervisory role of driving instructors is not sacrificed through the utilization of the developed training platform in driving schools.

- More than one participant can be trained simultaneously, while instructors have full centralized control on the training sessions. This ensures better time and effort utilization of interactive driving simulation in driving schools.

- An interactive simulation model for an autonomous driving system was implemented in this work. This model presents an example of potential complex systems that drivers must practice initially in safe virtual environments.

As potential future work, the capabilities of the developed training platform will be extended further so that the participating driving simulators can share the same virtual environment. The ability to create a virtual driving environment simultaneously accessed by two or more drivers allows a much closer approximation of reality, with its attendant risks and uncertainty [17]. Drivers have to react to each other and adapt their driving behavior accordingly. This increased training interactivity enables drivers to act in the same way as in real traffic environments. Moreover, driving instructors can participate more interactively by performing specific maneuvers with their simulated vehicles in 
order to subject drivers to sudden or unpredictable traffic situations. As participants of future traffic environments becoming more interconnected, networked driving simulation can be used to safely and efficiently learn various connected and cooperative vehicle technologies without overestimating their capabilities.

Acknowledgments: The presented work was carried out by the authors during the collaborative research and development activities at the Heinz Nixdorf Institute and the Fraunhofer Institute in Paderborn, Germany. No necessary special or external funding resources were acquired to produce or publish the research and development outcomes of this work. The authors acknowledge the valuable consultation and support of dSPACE GmbH, HELLA KGaA Hueck \& Co., Varroc Lighting Systems GmbH, Aerosoft GmbH, VDL Bus \& Coach GmbH, UNITY AG, Fahrerakademie Paderborn, Hainer driving school, and Ringhoff driving school.

Author Contributions: Kareem Abdelgawad analyzed the system requirements, developed the concept, built the prototype, and wrote the paper. Jürgen Gausemeier was consulted regarding the call for action and directed the research topic. Jörg Stöcklein, Michael Grafe, Jan Berssenbrügge, and Roman Domitrescu developed the visualization environment of the training platform. The latter five authors examined the work results and reviewed the paper.

Conflicts of Interest: All authors declare no conflict of interest.

\section{References}

1. Golias, J.; Yannis, G.; Antoniou, C. Classification of driver assistance systems according to their impact on road safety and traffic efficiency. J. Intell. Transp. Syst. 2002, 22, 179-196. [CrossRef]

2. Abdelgawad, K.; Gausemeier, J.; Dumitrescu, R.; Grafe, M.; Stöcklein, J.; Berssenbrügge, J. Networked Driving Simulation: Applications, State of the Art, and Design Considerations. Designs 2017, 1, 4. [CrossRef]

3. Abdelgawad, K.; Abdelkarim, M.; Hassan, B.; Grafe, M.; Gräßler, I. A Modular Architecture of a PC-based Driving Simulator for Advanced Driver Assistance Systems Development. In Proceedings of the 15th IEEE International Workshop on Research and Education in Mechatronics (REM2014), El Gouna, Egypt, 9-11 September 2014.

4. Abdelgawad, K.; Abdelkarim, M.; Hassan, H.; Grafe, M.; Gräßler, I. A Scalable Framework for Advanced Driver Assistance Systems Simulation. In Proceedings of the 6th International Conference on Advances in System Simulation (SIMUL2014), Nice, France, 12-16 October 2014.

5. Panou, M.C.; Bekiaris, E.D.; Touliou, A.A. ADAS module in driving simulation for training young drivers. In Proceedings of the IEEE 13th International Symposium on Intelligent Transportation Systems (ITSC), Funchal, Portugal, 19-22 September 2010; pp. 1582-1587.

6. Miller, D.; Sun, A.; Ju, W. Situation awareness with different levels of automation. In Proceedings of the IEEE International Conference on Systems, Man and Cybernetics (SMC), San Diego, CA, USA, 5-8 October 2014; pp. 688-693, INSPEC 14805632. [CrossRef]

7. Greenberg, J.; Blommer, M. Physical Fidelity of Driving Simulators. In Handbook of Driving Simulation for Engineering, Medicine, and Psychology; Fisher, D., Caird, J., Rizzo, M., Lee, J., Eds.; CRC Press Taylor \& Francis Group: Boca Raton, FL, USA, 2011; pp. 1-34.

8. Kaufmann, H.; Csisinko, M. Multiple Head Mounted Displays in Virtual and Augmented Reality Applications. Int. J. Virtual Real. 2006, 5, 1-10.

9. Gloria, A.; Bellotti, F.; Berta, R.; Lavagnino, E. Serious Games for Education and Training. Int. J. Serious Games 2014, 1, 100-105. [CrossRef]

10. Jazar, R.N. Vehicle Dynamics. In Vehicle Dynamics: Theory and Application, 2nd ed.; Jazar, R.N., Ed.; Springer Science + Business Media, LCC: New York, NY, USA, 2008; pp. 567-620.

11. Sivaji, V.V.; Sailaja, M. Adaptive Cruise Control Systems for Vehicle Modeling Using Stop and Go Manoeuvres. Int. J. Eng. Res. Appl. 2013, 3, 2453-2456.

12. Yi, K.; Cho, Y.; Lee, S.; Lee, J.; Ryoo, N. A Throttle/Brake Control Law for Vehicle Intelligent Cruise Control. In Proceedings of the Seoul 2000 FISITA World Automotive Congress, Seoul, Korea, 12-15 June 2000.

13. Abdelkarim, M.; Butz, T.; Moutchiho, A. A nonlinear path following controller for lateral vehicle guidance. In Fahrermodellierung in Wissenschaft und Wirtschaft, Fortschritt-Berichte VDI, Vol. 22, No. 35; VDI Verlag: Düsseldorf, Germany, 2013; pp. 135-145, ISBN 978-3-18-303522-9. 
14. Okuda, R.; Kajiwara, Y.; Terashima, K. A survey of technical trend of ADAS and autonomous driving. In Proceedings of the IEEE 13th International Symposium on VLSI Technology, Systems and Application (VLSI-TSA), Hsinchu, China, 21-25 April 2014; pp. 1-4.

15. Engen, T.; Lervag, L.-E.; Moen, T. Evaluation of IVIS/ADAS Using Driving Simulators. Comparing Performance Measures in Different Environments. In Proceedings of the European Transport Conference, Noordwijkerhout, The Netherlands, 5-7 October 2009; pp. 1-10.

16. Mao, Z.; Yan, X.; Zhang, H.; Wu, C. Driving Simulator Validation for Drivers' Speed Behavior. In Proceedings of the 2nd International Conference on Transportation Engineering, Chengdu, China, 25-27 July 2009; pp. 2887-2892.

17. Abdelgawad, K.; Gausemeier, J.; Trächtler, A.; Gausemeier, S.; Dumitrescu, R.; Berssenbrügge, J.; Stöcklein, J.; Grafe, M. An Application-Oriented Design Method for Networked Driving Simulation. Designs 2017, 1, 6. [CrossRef]

(C) 2017 by the authors. Licensee MDPI, Basel, Switzerland. This article is an open access article distributed under the terms and conditions of the Creative Commons Attribution (CC BY) license (http://creativecommons.org/licenses/by/4.0/). 